\title{
Prevención del alcoholismo e inversiones publicitarias en España: una lucha de David contra Goliath
}

\section{Alcoholism prevention and alcohol advertising investment in Spain: David versus Goliath}

Julio Montes-Santiago*; Marta Lado Castro-Rial ${ }^{* *}$
* Facultativo Especialista de Área. Servicio de Medicina Interna.

Complejo Hospitalario Universitario-Meixoeiro. Vigo.

${ }^{\star \star}$ MIR $5^{\circ}$ año. Servicio de Medicina Interna. Complejo Hospitalario Universitario-Meixoeiro.Vigo.

Enviar correspondencia a:

Julio Montes Santiago. Servicio de Medicina Interna.

Hospital Meixoeiro-CHUVI. 36200 Vigo (Pontevedra).

Teléfono: 98681111 ext. 11541

E-mail: julio.montes.santiago@sergas.es

\section{RESUMEN}

Objetivo: La publicidad de alcohol se correlaciona con su consumo, particularmente entre jóvenes. Estudiamos la evolución de las inversiones publicitarias globales y en bebidas alcohólicas en España durante el periodo 19952005.

Métodos: Se analizaron los informes anuales INFOADEX y otras fuentes informativas sobre la inversión publicitaria global y de bebidas alcohólicas en medios de comunicación convencionales (TV, radio, prensa escrita, cartelera externa e Internet), así como los gastos autonómicos en prevención. El estudio se subdividió entre los periodos 1995-2000 y 2001-2005.

Resultados: Entre 1995-2000 hubo incremento $(\Delta)$ en la publicidad alcoholera (de 268 a 347 millones $€$; $\Delta=29,5 \%$ ), pero disminución en el porcentaje de tal publicidad respecto a la global (de 7,6\% al 6,1\%). Entre 2001-2005 hubo $\Delta$ en las cantidades de publicidad (de 145 a 186 millones $€$; $\Delta=28,0 \%$ ) y del porcentaje con respecto a la global (del 2,7\% al 2,8\%). En 2001-2005 la inversión autonómica preventiva pasó de 22 a 52 millones $€(\Delta=136 \%)$.

Conclusiones: Los gastos publicitarios en alcohol permanecen altos en España, siendo los jóvenes objetivos preferentes. Aunque en aumento, son aún modestas las inversiones publicitarias en prevención. Se precisan medidas reguladoras para proteger a poblaciones susceptibles para su consumo inmoderado.

Palabras clave: alcohol, publicidad del alcohol, medios de comunicación.
ABSTRACT

Objective: Alcohol advertising correlates with consumption, particularly in young people. We studied the evolution of the amounts spent on alcoholic beverages advertising and on advertising as a whole in conventional media in Spain during the period 1995-2005.

Methods: We analyzed the amounts spent on advertising in total and on alcoholic beverages advertising by studying the annual INFOADEX Survey on Advertising Investment in Spain in conventional media (TV, radio, the press, billboards and Internet). The results were subdivided into the periods 1995-2000 and 2001-2005. Results: In the period 1995-2000 there was an increase $(\Delta)$ in alcoholic beverages advertising expenditure, from 268 to 347 million $€(\Delta=29.5 \%)$, but a decrease in its percentage of advertising as a whole (from $7.6 \%$ to $6.1 \%$ ). In the period 2001-2005 there was a rise in alcohol advertising expenditure from 145 to 186 million $€(\Delta=28.0 \%)$, and also in its percentage of total advertising (from $2.7 \%$ to 2.8\%). In 2001-2005, spending by Regional governments on preventive advertising increased from 22 to 52 million $€(\Delta=136 \%)$.

Conclusions: Alcohol advertising expenditure remains high in Spain, with young people as a primary target. In contrast, there is only modest investment in preventive advertising. Regulatory measures are necessary with a view to protecting populations especially susceptible to uncontrolled consumption.

Key words: alcohol, alcohol advertising, mass media. 


\section{INTRODUCCIÓN}

El 11 de agosto de 1956, a los 44 años, Jackson Pollock, el genial creador del expresionismo abstracto, quien 6 años antes asombrara y lograra hacer exclamar en la Bienal de Venecia a los críticos que "a su lado, el propio Picasso resulta un tranquilo conformista, un pintor del pasado", concluía sus dias estrellando su viejo Oldsmobile V-8 contra un árbol, en medio de una de sus nubes de alcohol. También moría una de sus acompañantes. Así acaba, en contraportada, la documentada biografía inspiradora de la película ganadora de un Oscar, Pollock, protagonizada y dirigida por Ed Harris'. El alcohol truncaba su vida en plena madurez, junto a las de otros personajes míticos de su generación: como Montgomery Clift, como Truman Capote... Como otros muchos personajes anónimos. En España, un tercio de las cerca de 3000 víctimas mortales anuales en accidentes de tráfico presentan niveles de alcoholemia $>0,3 \mathrm{~g} / \mathrm{dl}$.

Pero ello es sólo un aspecto del problema. Se ha asociado el consumo abusivo de alcohol con más de 60 enfermedades y se calcula que este es el responsable del $4 \%$ de la carga global mundial de enfermedad ${ }^{2,3}$. España ocupaba en 2003 el $6^{\circ}$ lugar mundial en consumo de alcohol. Esto se ha convertido en un asunto prioritario de Salud Pública y también económico ya que se estima en 3800 millones $€$ el coste global anual del consumo excesivo de alcohol en nuestro país ${ }^{4}$. Pero incluso más preocupante es la constatación de la elevada prevalencia de su consumo en jóvenes. Según la Encuesta Estatal sobre Uso de Drogas en los Estudiantes de Enseñanzas Secundarias (ESTUDES 2006-07) ${ }^{5}$, a pesar de observarse discretas mejorías respecto a estudios previos, el alcohol es la sustancia de consumo más extendida entre jóvenes de 14 a 18 años, habiéndolo probado alguna vez el $80 \%$, de ellos el $58 \%$ en el último mes. Más alarmante es que el $55 \%$ se han emborrachado alguna vez y el $22 \%$ de los estudiantes beben todos los fines de semana. Es especialmente frecuente el consumo "en atracón", o concentrado ("binge drinking" de los anglosajones) es decir, la ingesta de cantidades muy elevadas en cortos periodos de tiempo (5 o más bebidas en una sola ocasión) ${ }^{4-7}$. Además, en los últimos 12 meses el 22\% reconoce haber sido pasajero y el $10 \%$ conducido vehículos bajo sus efectos. Los conflictos, aún no resueltos, que ocasiona el fenómeno del "botellón" han llegado a considerarse tanto problema de salud como de orden público ${ }^{8}$.

Se ha demostrado que la publicidad del alcohol es, al menos, un factor moderado de incitación a probarlo entre los adolescentes 9 . La industria alcoholera destina inmensos recursos a su publicidad. En el año 2005 se insertaron en los diversos medios 452.251 anuncios de bebidas alcohólicas (1,6\% del global de anuncios y un $6 \%$ más que en 2000$)^{4}$, a menudo contraviniendo la normativa legal.

Este estudio analiza la evolución de las inversiones publicitarias en bebidas alcohólicas en los medios convencionales, su correspondencia con las de publicidad global y su comparación con las destinadas a prevención por los diferentes planes de drogas autonómicos en España, durante el periodo 1995-2005.

\section{MATERIAL Y MÉTODOS}

Se examinó en España durante el periodo 1995-2005 la inversión publicitaria total y la realizada por las compañias de bebidas alcohólicas en los medios llamados convencionales (TV, radio, prensa escrita, cartelera exterior e Internet). Para ello se analizaron los informes anuales INFOADEX sobre la Inversión Publicitaria en España sobre las materias reseñadas ${ }^{10}$. Además se investigó de forma más minuciosa el último informe publicado más detallado disponible (Informe INFOADEX 2006) ${ }^{106}$ para determinar la distribución preferida de la publicidad de productos alcohólicos según los medios de soporte. Debido a los desajustes causados por la entrada de España en la zona Euro, así como a los derivados de algunos cambios metodológicos en los Informes INFOADEX, que motivaron algunas inconsistencias en las cifras en 2001, se dividió el periodo de análisis en dos tramos -1995-2000 y 2001-2005-, para hacer más coherentes la comparaciones. Se utilizaron también aqueIlas fuentes secundarias de información que pudieran aportar datos relevantes sobre los temas estudiados ${ }^{11 a-c}$. Así mismo, se revisaron las publicaciones oficiales sobre las cantidades destinadas al apartado "Prevención", de los planes sobre las drogas de las Comunidades Autónomas (CCAA), así como las de prevención de accidentes realizadas por la Dirección General de Tráfico (DGT) ${ }^{12}$.

\section{RESULTADOS}

Como puede observarse en la figura 1, entre 1995 y 2000 hubo un incremento $(\Delta)$ en las cantidades de la publicidad en las bebidas alcohólicas (de 268 a 347 millones $€, \Delta=+29,5 \%$ ), aunque el porcentaje que supuso esta publicidad con respecto al global de publicidad se redujo en un $1,6 \%$ (del $7,6 \%$ al $6,1 \%)$. Las cifras de la publicidad convencional total pasaron
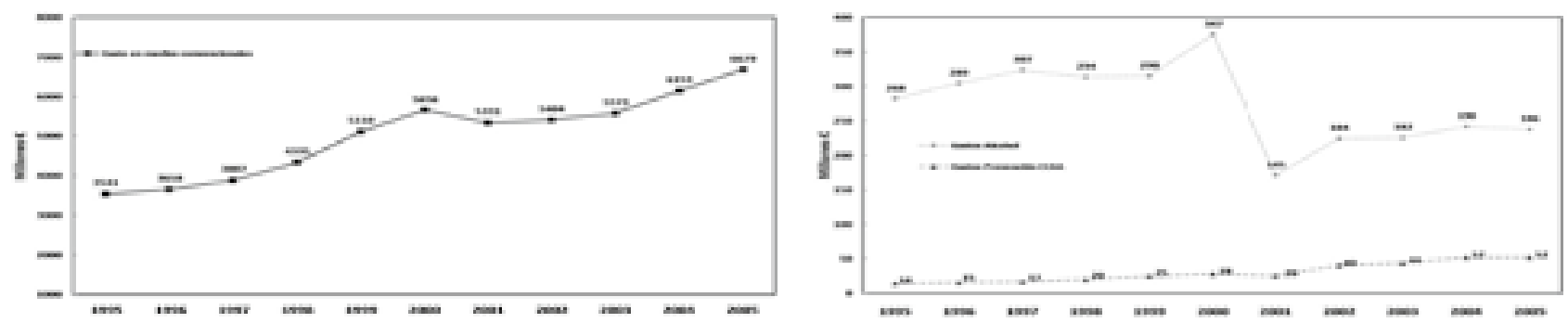

Figura 1. Evolución de las inversiones en publicidad en medios convencionales, en bebidas alcohólicas y en el área de prevención de las drogas de las Comunidades Autónomas. 
de 3550 a 5110 millones $€(\Delta=+59,7 \%)$. Entre 2001 y 2005 hubo un $\Delta$ en las cantidades dedicadas a la publicidad alcohólica (de 145 a 186 millones $€, \Delta=+28,0 \%$ ), y del porcentajes que supuso esta con relación a la global (de 2,7\% a 2,8\%, $\Delta=+0,1 \%)$. La publicidad convencional total pasó de 5331 a 6679 millones $€(\Delta=+25,3 \%)$. En diciembre-2007 la industria alcoholera gastó en TV 33,43 millones $€$ en campañas inductoras frente a 0,45 millones $€$ de las campañas preventivas $^{10 c}$. En cuanto al presupuesto para Prevención de las CCAA, aunque aumentó notablemente en el periodo 2001-2005 (de 22 a 52 millones $€, \Delta=+136 \%$ ), todavia queda muy lejos de los 186 millones $€$ invertidos por la industria alcoholera.

Con respecto a la elección de los soportes publicitarios (Tabla 1), la industria alcoholera se decanta preferentemente por los suplementos dominicales de la prensa escrita y la cartelera exterior (en esta última 3 de las principales marcas suponen el $10 \%$ del total publicitario). Por su parte, las compañias cerveceras hacen una decidida apuesta por el cine. En cuanto a las campañas de la DGT se concentraron, como parece lógico, en la radio (73\% del total de su presupuesto).

Tabla 1. Compañías anunciantes de bebidas alcohólicas y de prevención (Dirección General de Tráfico, DGT).

A) Situación dentro del ranking de los 100 primeros anunciantes en el global publicitario, cantidades invertidas y $\%$ de las mismas, respecto al global publicitario.

B) Situación dentro del ranking de los 10 primeros anunciantes de cada tipo de soporte de medio publicitario, cantidades invertidas y \% con respecto al total de inversión publicitaria en cada soporte publicitario.

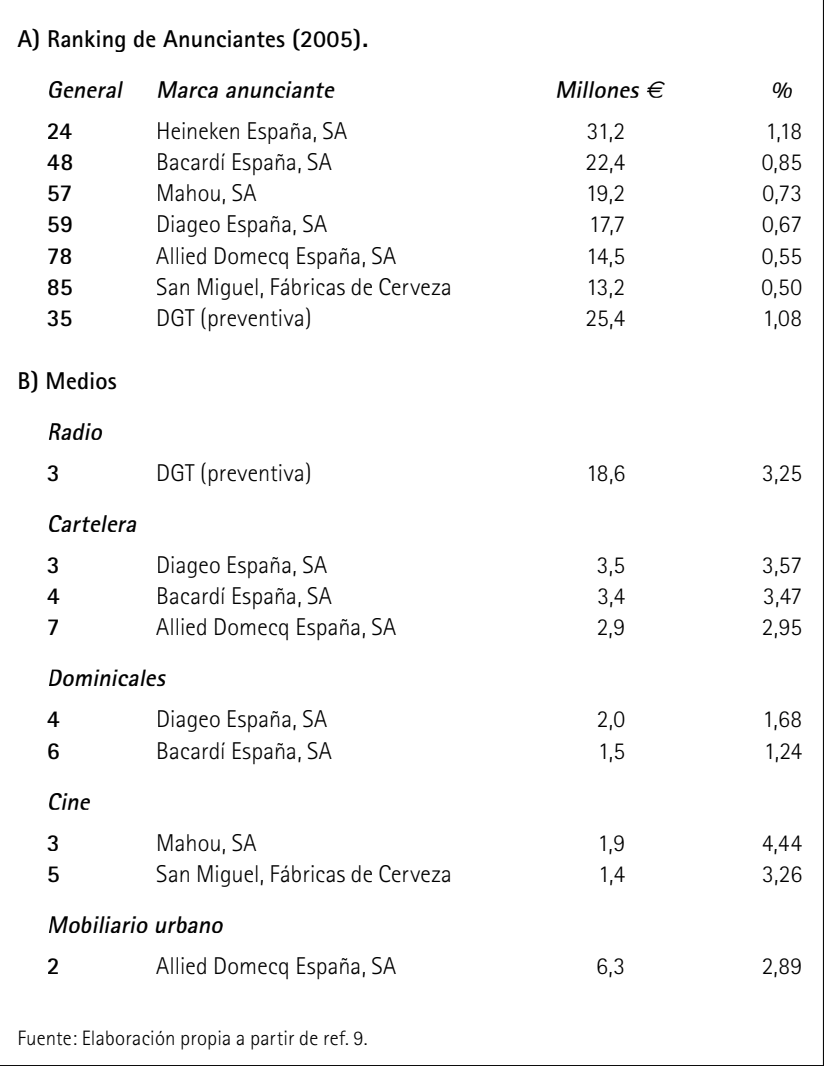

\section{DISCUSIÓN}

Resulta evidente que el alcohol es una gran asignatura pendiente en España ${ }^{13}$. Desde que en la primavera de 2006 y de modo sospechosamente concomitante, se convocaran "macrobotellones" en más de 20 ciudades españolas, tal tema no ha dejado de estar candente en el debate público. El punto culminante de tal polémica se alcanzó en febrero de 2007 con las encendidas disputas y posterior retirada de la ley reguladora del alcohol, debido a las presiones ejercidas por los sectores interesados. Han trascurrido desde entonces más de 2 años y, más allá de la cuestión de si tal problema se abordó de la forma más apropiada, y pese a la retirada final de la iniciativa legislativa, las discusiones suscitadas sirvieron para alcanzar algunos logros, quizá más modestos, aunque significativos. Entre ellos destacan la disminución de positividades de alcoholemias tanto en los controles aleatorios [de $4,2 \%(2003)$ a $1,9 \%(2008)]^{14}$, como en los accidentes mortales [de 34,7\% (2003) a 28,8\% (2006). El número anual de fallecidos en estos es ya inferior a 3000 (2741 en 2007 y 2181 en 2008) ${ }^{13}$, si bien este cifra infraestima en un 3,0-6,6\% el número real de fallecidos ${ }^{15}$.

Desde el punto de vista publicitario, se logró el compromiso por parte de la asociación $A E D E$, agrupadora de más de 90 cabeceras editoriales de diarios en España, para no exhibir anuncios de alcohol en las cabeceras y últimas páginas de los periódicos y un compromiso similar de no lucirlos ostensiblemente por parte de los vendedores de prensa. Así mismo, ciertas agencias publicitarias, clientes de la industria alcoholera, diseñaron campañas sobre el consumo responsable de alcohol, dirigidas fundamentalmente a jóvenes ${ }^{16 a-c}$. Esto constituyó un reconocimiento explícito de que ese colectivo era una diana preferente en las campañas publicitarias y supuso un cambio cualitativo en la postura hasta entonces mantenida por dicha industria, que negaba esa evidencia ${ }^{8}$. Así, diversos estudios habían mostrado que un $28 \%$ de los anuncios publicitarios de bebidas alcohólicas en TV, un 50\% en radio y un 33\% en prensa escrita tenían como destinatarios preferentes a los jóvenes ${ }^{4}$ 9, 16. Y ello a pesar de la observación de un creciente desplazamiento de esta inversión publicitaria desde tales medios clásicos a otros con menor selectividad de receptores -como la cartelera exterior (tabla 1)- o menos convencionales como Internet [en el cual el porcentaje de inversión publicitaria de esta industria pasó de 0,01\% (2001) a 0,1\% (2005)] .

No obstante, como nuestro estudio demuestra, los pequeños avances obtenidos no pueden empañar el hecho de la evidente desigualdad de recursos destinados a la publicidad de bebidas alcohólicas y los dedicados a prevención. Este análisis se ha focalizado en los medios convencionales, en los cuales, como acaba de referirse, ya se ha demostrado una primordial orientación a jóvenes. También puede citarse en los últimos tiempos a las mujeres como una diana preferente publicitaria ${ }^{18}$. Pero abundando aún más en el asunto, en un mes tan paradigmático como diciembre, sólo en TV en el año 2007, la inversión en campañas preventivo-educativas fue sólo el 1,3\% del destinado por la industria alcoholera en su publicidad $(0,45$ frente a 33,4 millones $€)^{11 c}$. 
El uso abusivo de alcohol se ha convertido hoy en una materia inquietante para la sociedad. La Asamblea Mundial de la Salud de la OMS, en su reunión de 2005, alertó de «la magnitud de los problemas de salud pública asociados al consumo nocivo de alcohol y las tendencias de consumo peligroso, en particular entre los jóvenes de muchos estados miembrosı". Y pidió a los mismos que elaborasen, aplicasen y evaluasen estrategias y programas eficaces para reducir las consecuencias sanitarias y sociales negativas de su consumo nocivo. Es evidente que en nuestro país debe seguirse reflexionando sobre esta cuestión. En un estudio internacional reciente de 30 paises, nuestro país ocupa un rango medio en cuanto a politicas regulatorias del consumo de alcohol. Sin embargo, sus precios, y por tanto la facilidad de acceso, son de los más bajos de los países analizados ${ }^{2}$. Este tema plantea desafíos a todos los niveles y exige a nuestra sociedad, al igual que se ha hecho en otros paises, debatir sobre el arbitrio de medidas equitativas que, preservando intereses legítimos, permitan evitar, sobre todo a sus miembros más jóvenes, los perjuicios asociados al consumo inmoderado $4,7,8,12,13,17$.

En conclusión, la inversión en la publicidad de bebidas alcohólicas permanece muy alta en España. En contraste, se invierten escasos recursos económicos en campañas preventivas, teniendo en cuenta que los jóvenes constituyen dianas preferentes de esta clase de productos. Se hace evidente la necesidad de instruir ciertas medidas regulatorias destinadas a la protección de las poblaciones especialmente susceptibles para el abuso de estas sustancias.

\section{REFERENCIAS}

1. Naifeh S, White Smith G. Jackson Pollock. Barcelona: Circe; 1991 [original, 1989].

2. Room R, Babor T, Rehm J. Alcohol and public health. Lancet 2005; 365: 519-30.

3. Brand DA, Saisana M, Rynn LA, Pennoni F, Lowenfels AB. Comparative analysis of alcohol control policies in 30 countries. PLos Med 2007; 4(4): e151. doi: 10.1371/journal. pmed.0040151 [consultado 15/11/08].

4. Ministerio de Sanidad y Consumo. Comisión Clínica de la Delegación del Gobierno para el Plan Nacional Sobre Drogas. Informe sobre Alcohol 2007. Disponible en: http://www.pnsd. msc.es/Categoria2/publica/pdf/InformeAlcohol.pdf. [consultado el 28/01/08].

5. Ministerio de Sanidad y Consumo. Secretaría General de Sanidad. Delegación del Gobierno para el Plan Nacional sobre Drogas. Informe de la encuesta estatal sobre uso de drogas en estudiantes de enseñanzas secundarias (Estudes) 2006-2007. Disponible en: http://www.pnsd.msc.es/Categoria2/observa/pdf/ Estudes_2006-2007.pdf [consultado el 31/01/08].

6. Farke W, Anderson P. El consumo de alcohol concentrado en Europa. Adicciones 2007; 19:333-40.
7. Calafat Far A. El abuso de alcohol de los jóvenes en España. Adicciones 2007; 19:217-24.

8. Montes-Santiago J, Álvarez Muñiz ML, Baz Lomba A. Alcohol y publicidad en la prensa escrita en España. An Med Interna (Madrid). 2007; 24: 109-12.

9. Gardfield CF, Chung PJ, Ratlur P. Alcohol advertising in magazines and adolescent readership. JAMA. 2003; 289: 2424-9.

10. Estudio INFOADEX de la Inversión publicitaria en España (Varios años, 1995-2007). Disponible en: http://www.infoadex. es/infoadex.asp. [consultado el 31-01-2008]. b) La inversión publicitaria en España. Anuario El País 2007. Madrid: Ediciones El Pais, 2007; 182-84.

11. Sánchez-Pardo L. Políticas reguladoras de la accesibilidad, disponibilidad y promoción de bebidas alcohólicas y tabaco. Boletín IDEA-Prevención 2003; 27: 88-101. b) Muñoz D. Se desata la guerra por las restricciones publicitarias al vino y la cerveza. Mercados del vino y la distribución 2007; 10: 8. c) Tudela A. 26 millones en anunciar coches y 2,2 en prevención de accidentes. Incluso las aseguradoras de coches gastaron más que el Estado en campañas de prevención.Disponible_en:_http://www.publico. es/dinero/034160/26/millones/anunciar/coche/2/2/prevencion/ accidentes [consultado el 05/01/08].

12. Ministerio de Sanidad y Consumo. Plan Nacional sobre Drogas. Memoria 2005. Disponible en: http://www.pmsd.msc.es/ Categorias2/publico/pdf.memo2005.pdf [consultado el 28/01/08].

13. Laso FJ, Pastor I. Alcoholismo: asignatura pendiente. Med Clin (Barc). 2004; 123: 538-9.

14. Ministerio del Interior. Dirección General de Tráfico. Balance de Seguridad Vial 2008. Disponible_en:http://www.dgt.es/was6/ portal/contenidos/documentos/prensa_campanas/notas_prensa/ notaprensa123.pdf [consultado el 12/04/08].

15. Pérez C, Cirera E, Borrell C, Plasencia A, en nombre del Grupo de Trabajo de la Sociedad Española de Epidemiología sobre la Medida del Impacto en la Salud de los Accidentes de Tráfico en España. Fallecidos a 30 dias por lesiones producidas por accidentes de tráfico en España. Gac Sanit. 2006; 20:1-7.

16. Anónimo La AEDE se compromete con Sanidad a rechazar la publicidad de alcohol en las portadas y contraportadas de sus diarios. Disponible en: http://www.elconfidencial.com/ cache/2007/06/11/3.html. [consultado el 11/06/07]. b) De Benito E. Sanidad recluta a los comerciantes en su lucha contra el consumo de alcohol por menores. Disponible en: http://www. elpais.com/articulo/sociedad/elpepisoc/20070614elpepisoc_1/Tes. [consultado el 14/06/07]. c) Anónimo. Diageo lanza una campaña de publicidad en TV para evitar el consumo abusivo de alcohol entre los jóvenes. Disponible en: http:// www.marketingnews.es/ Noticias/Granconsumo/20060724001 [consultado el 14/06/08].

17. Giesbrecht N. Desarrollos recientes sobre el consumo global de alcohol y consumos de alto riesgo: argumentos a favor de las intervenciones efectivas a nivel de población en Canadá. Adicciones 2008; 20: 207-220.

18. Quiles Soler MC. Publicidad de bebidas alcohólicas en revistas femeninas. Disponible_en_http://www.campusred.net/ forouniversitario/pdfs/Comunicaciones/PublicidadyMarketing/ mquiles.pdf [consultado el 28-01-2008]. 